\title{
In Response:
}

We would like to thank the author for their interest in our case report and their supportive comments. It is encouraging that they were able to use pulsed radiofrequency (PRF) to successfully treat meralgia paresthetica in 2 additional patients. In the cases of the 52-year-old and 80-year-old patients, the duration of pain relief reported by the authors is noteworthy. In our own patient, follow-up at the one-year point revealed that she remained pain free, had been able to exercise, lost 75 pounds, and had an enhanced social and professional life (1). Each of these respective cases emphasizes the potential long-term benefit of this non-neurodestructive analgesic and anti-nociceptive modality in managing difficult, chronic pain scenarios.

As pointed out by the author, although this treatment can be considered low risk, care should be taken to prevent injury to nearby anatomical structures. Dalmau-Carolà discussed what she perceives could be a potential complication, namely intestinal perforation. Certainly, our patient was apprised in detail about this potential. Although not recommended, we suggest that a single unintentional passage through the colon with a 20-gauge needle in a non-anticoagulated patient may not have grave sequelae. To emphasize this point, we consider Brandt et al's (2) reports of biopsies of the pancreas. They described 66 separate intentional passes through the gastrointestinal tract using 1821 gauge needles with no complications (2).
Additionally, Dalmau-Carolà reported inserting the needle at a more caudad location than we did, which highlights the interaction between both fluoroscopic and functional anatomy. It also underscores the variability presented by each individual patient. Hospodar et al (3) claim in their anatomical study of 50 cadavers, various jaunts coursed by the lateral femoral cutaneous nerve (LFCN). They claim the course of the LFCN to be in the range between 3 to $46 \mathrm{~mm}$ medial to the anterior superior iliac spine (ASIS). They further maintain in 10 percent of the population the LFCN traverses lateral to the ASIS. It is with this anatomical insight that we suggest that eliciting concordance of pain is of the utmost importance when determining the point of entry despite the possible risks including that of the aforementioned colonic perforation.

The anecdotal findings presented by us and by the present author provide a framework and basis for conducting well-designed studies to assess the use of PRF under controlled scientific conditions.

\author{
Kenneth D. Candido, MD \\ Department of Anesthesiology \\ and Pain Management \\ Advocate Illinois Masonic Medical Center \\ 836 W. Wellington Avenue \\ Chicago, IL 60657 \\ E-mail: kdcandido@yahoo.com
}


Cyril N Philip, MD

Department of Anesthesiology and Pain Management

Advocate Illinois Masonic Medical Center

836 W. Wellington Avenue

Chicago, IL 60657.

George J. Crystal, PhD

Department of Anesthesiology and Pain Management
Advocate Illinois Masonic Medical Center

836 W. Wellington Avenue

Chicago, IL 60657.

Ninos Joseph, BS

Advocate Illinois Masonic Medical Center

Department of Anesthesiology

Division of Pain Management

Chicago, IL

\section{References}

1. Philip CN, Candido KD, Joseph NJ, Crystal GJ. Successful treatment of meralgia parethestica with pulsed radiofrequency of the lateral femoral cutaneous nerve. Pain Physician 2009; 12:881

2. Brandt KR, Charboneau JW, Stephens DH, Welch TJ, Goellner JR. CT and US guided biopsy of the pancreas. Radiology 1993; 187:99-104.
3. Hospodar PP, Ashman ES, Traub JA. Anatomic study of the lateral femoral cutaneous nerve with respect to the ilioinguinal surgical dissection. Journal of Orthopaedic Trauma 1999; 13:17-19. 